\title{
Approximation Functionals and Their Application
}

\author{
A.Yuri K.DEM’YanOVich, TATJANA A.SAFONOVA, ANDREY N.TEREKHOV \\ Saint Petersburg State University, RUSSIA
}

\begin{abstract}
The purpose of this work is to obtain local estimates, and to calculate the constants of approximation of smooth functions. To achieve this goal, the trajectory of functionals, the concept of an approximation families of functionals, the tape of the trajectory of functionals and the width of the tape are considered. For this, the concepts of approximation functionals and their trajectories in the conjugate space are introduced, and the concepts of the trajectory tape and the tape width at a given point are discussed. The resulting overall evaluation of the approximation is applied to multidimensional cases, namely, to approximations by the Courant and Zlamal elements.
\end{abstract}

Keywords - finite element method, approximation functionals, evaluations of approximation

Received: April 29, 2021. Revised: August 12, 2021. Accepted: September 6, 2021. Published: September 21, 2021.

\section{Introduction}

Modeling various phenomena requires constant development approximation apparatus. This is due not only to the emergence of new tasks, but also with the need to clarify solutions that were earlier assigned to tasks. Using approximations to solve various problems are considered in works [10], [15], [21], [23]-[27]. In this regard, there has been a relentless development of spline methods for decades (see [1], [7], [9], [11], [16]). A similar pace has a constant development and deepening of the finite element methods (see [2]-[6], [12], [18]-[20]). It is easy to see that in all cases, the basis is a certain approximation apparatus. To construct an approximation apparatus which is required for various phenomena, the approximation relations are applied (see [2]-[3], [5], [9], [19], [21]). The mentioned relations are considered as a system of equations for the unambiguous determination coordinate functions in some subdivision of their area definitions.

The first works in this direction were works by S.G. Mikhlin and J. Strang (see [2]-[3]). In what follows, the approximation ratios were considered for units of various domains up to arbitrary differentiable manifolds (see [9], [19]).

The approximation relations turned out to be a convenient tool for constructing a set of new approximations, called local ones. This, of course, includes almost all finite element approximations and splines. The use of approximation relations led to the construction of wavelet expansions of a new type, thanks to the systems of functionals generated by them that are biorthogonal to coordinate functions. All this can be done in the most general situations (nonuniform grids, cell subdivisions, differentiable manifolds, see [27]). They make it possible to construct a logically coherent theory of nesting and smoothness of spaces of local functions. Finally, the need to approximate functions depending on a point by a sufficiently arbitrary surfaces, led to the development of the theory of approximation functions defined on a differentiable manifold (see [8] - [9], [13] [14], [17], [22]). The entropy estimate on a manifold was considered at work [13]. The paper [17] was devoted to the local linear regression on the manifold. Approximations using moving projections were investigated in the article [22]. In works [8] - [9], [14], approximations of functions defined on a manifold and established global estimates of the rate of convergence of the mentioned approximations were built. Most of the well-known works (see [1] -[27]) are to one degree or another related to the issues of approximation. This applies to works on the method of finite elements, on the method of meshes and on the wavelet method. They illustrate the urgent need to develop a common approach to approximation that would be a unifying factor in research. It is desirable that this approach gives local estimates indicating the constants, and also contains the ability to take into account changes in the characteristics of the function being approximated. If the approximated function is characterized by transitions from fast change to slow change and vice versa, then local estimates of the approximation and acceptable estimates of the approximation constants are required to improve the quality of their applications. In some works, the approximation constants are calculated (see, for example, [2]), but in most cases the approximation constants are not provided with effective estimates (see [3] -[24], [26] -[27]).

The proposed approach serves to overcome this disadvantage. This approach is characterized by the introduction and use of new mathematical objects, namely, the approximation trajectory of functionals, the concept of a tape for a trajectory and the width of this tape.

The aim of the work is to develop a general approach to constructing an approximation apparatus suitable for the local adaptive approximation of functions whose characteristics change rapidly when passing from one region of variation of independent variables to another (i.e., for functions with rapidly changing characteristics). The proposed approach allows one to obtain estimates of local approximation with the calculation of constants in the general situation and to develop adaptive approximation schemes that take into account the rate of change of the approximated function. 
The methods considered in this paper do not require (very laborious) processes for cutting the region into a finite number of parts followed by gluing the approximations obtained on each of the mentioned parts. Therefore the discussed method can be applied to functions defined on a differentiable manifold. The resulting overall evaluation of the approximation is applied to multidimensional cases, namely, to approximations by the Courant and Zlamal elements.

\section{Approximation Relations}

Let $\boldsymbol{M}$ be a closed set in the Euclidean space $R^{n}$. Let $\mathrm{C}(\boldsymbol{M})$ be the linear normed space of the continuous functions $w(\xi), \quad \xi \in \boldsymbol{M}$, with norm $\|w\|_{\mathrm{C}(\boldsymbol{M})}=\max _{\xi \in \boldsymbol{M}}|u(\xi)|$. Consider an open domain $\Omega$ in the space $R^{n}$.

Discussing the linear functionals $g$ in the normalized space $C(\bar{\Omega})$, we will consider their supports as closed sets. Typically, the action of a linear functional $g \in C^{*}(\bar{\Omega})$ to a function $w(\xi), \xi \in \bar{\Omega}, \mathrm{w} \in C(\bar{\Omega})$, is written as $\langle g, w(\cdot)\rangle$ or as $\langle g, w\rangle$, and in some cases --- in the form

$<g, w(\xi)>$. By $\left.w(\xi)\right|_{\xi \in D}$ (or $\left.w\right|_{D}$ ) we denote the restriction of the function $w(\xi)$ to the set $D, D \subset \bar{\Omega}$. Let us agree to assume that $\left\langle g,\left.w\right|_{\text {supp } g}\right\rangle=\langle g, w\rangle$. Let for each $t \in \bar{\Omega}$ in the space $C(\bar{\Omega})$ a linear functional $f(t)$

be defined such that $\operatorname{supp} f(t) \subset \bar{\Omega}$. The set $f=\{f(t) \mid \forall t \in \bar{\Omega}\}$ is called a trajectory in the space $C^{*}(\bar{\Omega})$.

Let $[x, y]$ be the straight line segment connecting points $x$ and $y$ of the space $R^{n}$, i.e. $[x, y]=\{z \mid \mathrm{z}=\theta x+(1-$ $\theta) y \forall \theta \in[0,1]\}$. Each $t \in \bar{\Omega}$ we associate with the set $S_{t}=$ $\{[t, \xi] \mid \xi \epsilon \operatorname{supp} f(t)\}$. The aggregate $\mathrm{B}(f)=\left\{S_{t} \mid \forall t \epsilon \bar{\Omega}\right\}$ is called by the tape of the trajectory for $f$, and the number

$$
h_{t}(f)=\max _{\xi \in S_{t}}\|\xi-t\|_{R^{n}}
$$

is called the width of the tape $\mathrm{B}(f)$ at the point $t$. The tape $\mathrm{B}(f)$ is called regular tape in the subregion $\varpi \subset \Omega$, if the condition

$$
S_{t} \subset \varpi \forall t \epsilon \varpi
$$

is fulfilled.

It is evident that the tape $\mathrm{B}(f)$ is regular in $\varpi$, if $\varpi=\Omega$ and $\Omega$ is the convex region.

If $w \in C(\bar{\Omega})$ then we have

$$
<f(t), w(\cdot)>=<f(t),\left.w(\xi)\right|_{\xi \in \operatorname{supp} f(t)}>
$$

Therefore

$$
|<f(t), w(\cdot)>| \leq \|\left. f(t)\right|_{C^{*}(\overline{\operatorname{supp} f(t)})}|| w||_{C(\overline{\operatorname{supp} f(t)})} .
$$

Suppose that the property

$$
<f(t),(\cdot-t)^{\alpha}>=\delta_{0, \alpha} \forall t \epsilon \bar{\Omega}, \quad|\alpha| \leq m,
$$

is fulfilled.

Relations (4) are called approximation relations, and functionals $f(t)$ are called approximation functionals.

Approximation relations (4) can be rewritten in the equivalent form

$$
<f(t), \varphi_{\alpha}(\cdot)>=\varphi_{\alpha}(t) \forall t \epsilon \bar{\Omega}, \quad|\alpha| \leq m,
$$

where $\varphi_{\alpha}(t)=t^{\alpha}$.

By Taylor's formula for a function $u \epsilon C^{m+1}(\bar{\Omega})$ we have

$u(\xi)=\sum_{|\alpha| \leq m} \frac{1}{\alpha !} u^{(\alpha)}(\mathrm{t})(\xi-t)^{\alpha}+\boldsymbol{R}_{\boldsymbol{m}}(\xi, t)$

where $[t, \xi] \subset \Omega$,

$$
\begin{aligned}
& \boldsymbol{R}_{\boldsymbol{m}}(\xi, t)=(m+1) \times \\
& \times \int_{0}^{1}(1-\tau)^{m} \sum_{|\alpha|=m+1} \frac{(\xi-t)^{\alpha}}{\alpha !} u^{(\alpha)}(\mathrm{t}+\tau(\xi-\mathrm{t})) \mathrm{d} \tau
\end{aligned}
$$

If the subdomain $\varpi \subset \bar{\Omega}$ and $[t, \xi] \subset \varpi$, then relation (7) implies the estimate

$$
\left|\boldsymbol{R}_{\boldsymbol{m}}(\xi, t)\right| \leq C_{m}^{\varpi}(u)\|\xi-t\|_{R^{n}}^{m+1},
$$

where

$$
C_{m}^{\varpi}(u)=(m+1) \sum_{|\alpha|=m+1} \frac{\left\|u^{(\alpha)}\right\|_{C(\varpi)}}{\alpha !}
$$

Applying $f(t)$ to relation (6), we obtain

$$
\begin{gathered}
<f(t), u(\cdot)>=\sum_{|\alpha| \leq m} \frac{1}{\alpha !} u^{(\alpha)}(\mathrm{t})<f(t),(\cdot-t)^{\alpha}>+ \\
+<f(t), \boldsymbol{R}_{\boldsymbol{m}}(\cdot, t)>.
\end{gathered}
$$

Using assumption (4), from relation (10) we find

$$
<f(t), u(\cdot)>=u(t)+<f(t), \boldsymbol{R}_{\boldsymbol{m}}(\cdot, t)>
$$

Assuming

$$
\tilde{u}(t)=<f(t), u(\cdot)>
$$

we rewrite formula (11) in the form

$$
\tilde{u}(t)-u(t)=<f(t), \boldsymbol{R}_{\boldsymbol{m}}(\cdot, t)>
$$

By formula (3) we have

$$
\begin{gathered}
\left|\boldsymbol{R}_{\boldsymbol{m}}(\cdot, t)\right| \leq \\
\leq\|f(t)\|_{C^{*}(\overline{\operatorname{supp} f(t)})}\left\|\boldsymbol{R}_{\boldsymbol{m}}(\cdot, t)\right\|_{C(\overline{\operatorname{supp} f(t)})}
\end{gathered}
$$

Consider the second factor on the right-hand side in inequality (14). We have

$$
\left\|\boldsymbol{R}_{\boldsymbol{m}}(\cdot, t)\right\|_{C(\overline{\operatorname{supp} f(t)})}=\max _{\xi \in \overline{\operatorname{supp} f(t)}}\left|\boldsymbol{R}_{\boldsymbol{m}}(\xi, t)\right| .
$$

If relation (3) is satisfied then by inequality (8) we find

$$
\begin{gathered}
\max _{\xi \epsilon \operatorname{supp} f(t)}\left|\boldsymbol{R}_{\boldsymbol{m}}(\xi, t)\right| \leq \\
\leq C_{m}^{\varpi}(u) \max _{\xi \epsilon \overline{\operatorname{supp} f(t)}} \| \xi-\left.t\right|_{R^{n}} ^{m+1} \forall t \in \varpi .
\end{gathered}
$$


Theorem 1. Let $f=\{f(t) \mid \forall t \in \bar{\Omega}\}$ be trajectory in space $C^{*}(\bar{\Omega})$. Let relation (4) and condition (3) be fulfilled. If $u \in C^{m+1}(\bar{\Omega})$ then the inequality

$|\tilde{u}(t)-u(t)| \leq$
$\leq C_{m}^{\varpi}(u)|| f(t) \|_{C^{*}(\operatorname{supp} f(t))}\left[h_{t}(f)\right]_{R^{n}}^{m+1} \forall t \in \varpi$

is right.

Here the constant $C_{m}^{\varpi}(u)$ is defined by formula (9), and the value $h_{t}(f)$ is indicated in relation (1).

Proof. From formulas (13) and (14) we obtain

$\widetilde{u}(t)-u(t)\left|\leq\|f(t)\|_{C^{*}(\overline{\operatorname{supp} f(t)})}\left\|\boldsymbol{R}_{\boldsymbol{m}}(\cdot, t)\right\|\right|_{C(\overline{\operatorname{supp} f(t)})}$.

Using relation (15), we derive

$\widetilde{u}(t)-u(t) \mid \leq\|f(t)\|_{C^{*}(\operatorname{supp} f(t))} \times$

$\times C_{m}^{\varpi}(u) \max _{\xi \in \operatorname{supp} f(t)}\|\xi-t\|_{R^{n}}^{m+1} \forall t \in \varpi$.

Using notation (1) in formula (17), we obtain relation (16). This completes the proof.

\section{Piecewise linear approximation}

In this section we discuss the approach to the Courant approximations. In addition to the Cartesian coordinates of the point $t$ in the Euclidean space $R^{n}$ the barycentric coordinates are needed in what follows. Let the points $x_{1}, x_{2}, \ldots, x_{n+1}$ of the space $R^{n} \quad$ serve as vertices of the nondegenerate simplex $T$, which is an open set (thus the simplex $T$ is homeomorphic to an open ball).

Consider $n+1$-component vector function (column) $\varphi(t)=\left(1, t^{(1)}, t^{(2)}, \ldots, t^{(n)}\right)$. In what follows, all considered vectors are discussed as column vectors (note that to save space we often represent a column vector as a row).

The barycentric coordinates of the point $\mathrm{t}=\left(t^{(1)}, \ldots, t^{(n)}\right)$ in the space $R^{n}$ is called an ordered collection of numbers

$\lambda^{T}(t)=\left(\lambda_{1}^{T}(t), \lambda_{2}^{T}(t), \ldots, \lambda_{n+1}^{T}(t)\right)$ defined by relations

$$
\lambda_{j}^{T}(t)=\frac{\operatorname{det}\left(\varphi\left(x_{1}\right), \varphi\left(x_{2}\right), \ldots,{ }^{\prime} j \varphi(t), \ldots, \varphi\left(x_{n+1}\right)\right)}{\operatorname{det}\left(\varphi\left(x_{1}\right), \varphi\left(x_{2}\right), \ldots, \varphi\left(x_{n+1}\right)\right)} .
$$

Here the symbol ${ }^{\prime} j$ means that the $\mathrm{j}$-th column of the determinant is replaced by the column $\varphi(t), j=1, \ldots, n+1$.

As it is known (see, for example, [4], p.55) the barycentric coordinates possess the properties

$$
\begin{gathered}
\lambda_{j}^{T}\left(x_{i}\right)=\delta_{i, j}, \quad \sum_{j=1}^{n+1} \lambda_{j}^{T}(t)=1 \quad \forall t \in R^{n}, \\
0<\lambda_{j}^{T}(t)<1 \quad \forall j=1,2, \ldots, n+1 \quad<=>\quad t \in T .
\end{gathered}
$$

Let the domain $\Omega$ have a piecewise linear boundary. Consider the simplicial subdivision $\mathbf{T}$. Lots of $T^{0}=$ $\left\{x_{i} \mid i \epsilon J\right\}$ vertices $x_{i}$ of this subdivision is called a zerodimensional skeleton; here $J$ is a finite set of indices. Let $x_{j}=\left(x_{j}^{(1)}, x_{j}^{(2)}, \ldots, x_{j}^{(n)}\right)$ be a vector, composed of cartesian coordinates of the vertex $x_{j}$. Vertices are also called nodes of the unit under consideration. By symbols $T$ we denote an open $n$-dimensional simplex of the subdivision $\mathbf{T}$.

Select the vertex $x_{i}$ of this subdivision and consider the closure of the union of simplices incident to a given vertex. The closure is called the body of the barycentric star for the vertex $x_{i}$. Let's denote it by $Z_{i}$. The barycentric star $Z_{i}$ is the support of coordinate function $\omega_{i}(t)$ of R. Courant's approximation. The function $\omega_{i}(t)$ can be determined by the approximation relations

$\sum_{x_{i} \in \bar{T}} \varphi\left(x_{i}\right) \omega_{i}(t)=\varphi(t), t \in T, \operatorname{supp} \omega_{j}(t) \subset Z_{j}, j \epsilon J$.

Since the simplex $T$ is nondegenerate, the system $\left\{\varphi\left(x_{i}\right)\right\}_{x_{i} \in \bar{T}}$ is a linearly independent system of vectors.

Without loss of generality, we assume that the vertices $x_{i}$ of the simplex $T$ are numbered by $1,2, \ldots, n+1$. Then the solution to the system (20) can be represented by Cramer's formulas in the form

$$
\omega_{j}(t)=\frac{\operatorname{det}\left(\varphi\left(x_{1}\right), \varphi\left(x_{2}\right), \ldots,,^{\prime j} \varphi(t), \ldots, \varphi\left(x_{n+1}\right)\right)}{\operatorname{det}\left(\varphi\left(x_{1}\right), \varphi\left(x_{2}\right), \ldots, \varphi\left(x_{n+1}\right)\right)} .
$$

Formulas (18) -- (21) show that $\omega_{j}(t)$ are barycentric coordinates of the point $t$ relative to the vertices $x_{j}$ of the simplex $T$. Thus

$$
\omega_{j}(t) \equiv \lambda_{j}^{T}(t) \quad \forall j \epsilon\{1,2, \ldots, n+1\}, t \in T .
$$

As a trajectory $f$ in the space $C^{*}(\bar{\Omega}) \quad$ consider the family of functionals $f(t), t \in \bar{\Omega}$, defined by the formula

$$
<f(t), u>=\sum_{x_{i} \in \bar{T}} u\left(x_{i}\right) \omega_{i}(t), \quad t \in \bar{T},
$$

It follows from formulas (1) and (23) that the width of the tape of the considered trajectory $f$ at the point $t \in \bar{T}$ satisfies the inequality

$$
h_{t}(f) \leq \operatorname{diam} T,
$$

where $\operatorname{diam} T=\sup _{\xi, t \epsilon T}\|\xi-t\|_{R^{n}}$.

Noting that approximation relations (20) can be rewritten in the form

$$
\begin{gathered}
\sum_{x_{i} \in \bar{T}} x_{i}^{\alpha} \omega_{i}(t)=t^{\alpha}, t \in T,|\alpha| \leq 1, \\
\operatorname{supp} \omega_{j}(t) \subset Z_{j}, \quad j \in J,
\end{gathered}
$$

we use Theorem 1 for $m=1$.

Theorem 2. Consider the trajectory $f$, defined by formula (23). For function $u$ from the class $C^{2}(\bar{\Omega})$ construct an approximation $\tilde{u}(t)$ by the formula 


$$
\tilde{u}(t)=\sum_{x_{i} \in \bar{T}} u\left(x_{i}\right) \omega_{i}(t), \quad t \epsilon \bar{T}, \quad T \epsilon \boldsymbol{T} . \quad \text { (26) }
$$

where the functions $\omega_{j}(t)$ are obtained from the approximation relations (20). Then the inequality

$$
\begin{aligned}
& |\tilde{u}(t)-u(t)| \leq \\
& \quad \leq 2 \sum_{|\alpha|=2}\left\|u^{(\alpha)}\right\|_{C(\bar{T})}(\operatorname{diam} T)^{2}
\end{aligned}
$$

is right.

Proof. Obviously, the conditions of Theorem 1 are satisfied for $m=1$. By formula (16) we have

$|\tilde{u}(t)-u(t)| \leq$

$\leq C_{1}^{\varpi}(u)\|f(t)\|_{C^{*}(\overline{\operatorname{supp} f(t)})}\left[h_{t}(f)\right]_{R^{n}}^{2} \quad \forall t \in \varpi$

Let $\varpi$ be simplex $T$. According to formula (9), we have

$$
C_{1}^{T}(u) \leq 2 \sum_{|\alpha|=2}\left\|u^{(\alpha)}\right\|_{C(\bar{T})} .
$$

Let's turn to the estimate $\|f(t)\|_{C^{*}(\overline{\operatorname{supp} f(t)})}$.

From the ratio (23) we have

$$
|<f(t), u>| \leq \max _{x_{i} \in \bar{T}}\left|u\left(x_{i}\right)\right| \sum_{x_{i} \in \bar{T}}\left|\omega_{i}(t)\right|, \quad t \in T .
$$

Using the non-negativity of the functions $\omega_{i}(t)$ and taking into account relation (25) for $\alpha=(0,0, \ldots, 0)$, we have

$$
\sum_{x_{i} \in \bar{T}}\left|\omega_{i}(t)\right|=1, \quad t \in T \text {. }
$$

From (30) and (31) we obtain

$$
\|f(t)\|_{C^{*}(\overline{\operatorname{supp} f(t)})} \leq 1 .
$$

Using formulas (24), (29), and (32) in relation (28), we have inequality (27). This completes the proof.

\section{Zlamal's approximation}

Similarly to the previous one, consider some general

Zlamal's approximation (see [4], p. 59).

Let $T \epsilon \mathbf{T}$ be simplex with vertices $x_{1}, x_{2}, \ldots, x_{n+1}$. Consider points $x_{i j}=\theta_{i j} x_{i}+\left(1-\theta_{i j}\right)$ on its edges $\left[x_{i}, x_{j}\right]$, $\mathrm{i}<j$, where $\theta_{i j} \in(0,1)$. It is clear to see that the polynomial $P^{T}(t)$ of the second order from $n$ variables $t=\left(t_{1}, \ldots, t_{n}\right)$,

$$
\begin{aligned}
P^{T}(t)= & \sum_{i=1}^{n+1} \lambda_{i}^{T}(t)\left(2 \lambda_{i}^{T}(t)-1\right) v_{i}+ \\
& +\sum_{1 \leq i<j \leq n+1} \frac{\lambda_{i}^{T}(t) \lambda_{j}^{T}(t)}{\theta_{i j}\left(1-\theta_{i j}\right)} v_{i j}
\end{aligned}
$$

is the solution of the interpolation problem

$$
\begin{gathered}
P^{T}\left(x_{i}\right)=v_{i}, \\
P^{T}\left(x_{i j}\right)=v_{i j}, \quad 1 \leq i<j \leq n+1 .
\end{gathered}
$$

Thus, we can assume that the numbers $v_{i}$ are given at the vertices $x_{i}$, and the numbers $v_{i j}$ are given at the selected points $x_{i j}$ edges of simplex $T$, where $1 \leq i<j \leq n+1$.

Now consider the interpolation problem defined on simplicial subdivision $\mathbf{T}$. Let there be some numbering of all vertices $x_{i}$ of the zero-dimensional skeleton $T^{0}, i \in\{1, \ldots, M\}$. We will associate indexing of edges $l$ in the one-dimensional skeleton $T^{1}$ with this numbering. To do this, we use ordered pairs of natural numbers $(j, k), j<k, j, k \in\{1, \ldots, M\}$, and put

$l_{j k}=\left[x_{j}, x_{k}\right]$. To each edge $l_{j k}, j<k$, we assign a number $\theta_{j k} \epsilon(0,1)$ and put $x_{j k}=\theta_{j k} x_{j}+\left(1-\theta_{j k}\right) x_{k}$. Consider an interpolation problem

$$
\begin{aligned}
& P\left(x_{i}\right)=v_{i}, \quad x_{i} \in T^{0}, \\
& P\left(x_{j k}\right)=v_{j k}, \quad l_{j k} \in T^{1},
\end{aligned}
$$

where $v_{i}$ and $v_{j k}$ are given numbers, $i, j, k \in\{1,2, \ldots, M\}, j<k$.

Solving problem (36) -- (37) in the class of piecewise polynomial functions of the second degree, similar to the previous one (see formulas (33) -- (35)) we arrive at a piecewise polynomial continuous functions $P(t)$,

$$
\begin{array}{r}
P(t)=\sum_{i=1}^{n+1} \lambda_{i}^{T}(t)\left(2 \lambda_{i}^{T}(t)-1\right) v_{i}+ \\
+\sum_{1 \leq i<j \leq n+1} \frac{\lambda_{i}^{T}(t) \lambda_{j}^{T}(t)}{\theta_{i j}\left(1-\theta_{i j}\right)} v_{i j}, \quad \forall t \in T \quad \forall T \epsilon \mathbf{T} .
\end{array}
$$

It is easy to distinguish the coordinate functions of this approximation. We fix the vertex $x_{s} \in T^{0}$ and in problem (36) -- (37) put $v_{i}=\delta_{i s}, v_{j k}=0$. As a result we get

$P_{s}^{T}(t)=\lambda_{s}^{T}(t)\left(2 \lambda_{s}^{T}(t)-1\right) \quad \forall t \in T \quad \forall T \epsilon \mathbf{T}$.

Hence it is seen that the coordinate function corresponding to the vertex $x_{s} \in T^{0}$ has the form

$$
\omega_{s}(t)=\left\{\begin{array}{c}
P_{s}^{T}(t) \quad \forall t \in T \quad \forall T \epsilon Z_{s} \\
0 \quad \forall t \in T \quad \forall T \epsilon \bar{\Omega} \backslash Z_{s}
\end{array} .\right.
$$

The coordinate function, corresponding to $x_{p q}, p<q$, can be found from the interpolation problem (37) -- (38), if $l_{p q} \in T^{1}$ and $v_{i}=0, v_{j k}=\delta_{j p} \delta_{k q}$. We have

$$
P_{p q}^{T}(t)=\frac{\lambda_{p}^{T}(t) \lambda_{q}^{T}(t)}{\theta_{p q}\left(1-\theta_{p q}\right)} \quad \mathrm{t} \in \bar{T}, x_{p q} \in \bar{T}
$$

From this we obtain the coordinate function

$\omega_{p q}(t)=\left\{\begin{array}{rl}P_{p q}^{T}(t) & \text { for } t \epsilon \bar{T}, T \subset Y_{p q}, \\ 0 & \text { for } t \epsilon \bar{\Omega} \backslash Y_{p q}\end{array}\right.$,

where $Y_{p q}$ is the union of all closed simplices, containing an edge $l_{p q}, Y_{p q}=\bigcup_{l_{p q} \in \bar{T}} \bar{T}$. 
Lemma 1. The next relations

$$
\begin{gathered}
\left|\omega_{s}(t)\right| \leq 1, \\
0 \leq \omega_{p q}(t) \leq\left[\theta_{p q}\left(1-\theta_{p q}\right)\right]^{-1}
\end{gathered}
$$

are right.

Proof. Taking into account properties (19) and inequality $\tau(2 \tau-1) \leq 1 \forall \tau \epsilon[0,1], \quad$ by the relations (39) -- (40) we obtain (43). Inequality (44) follows from inequality (19) and formulas (41) -- (42).

Consider the trajectory $f$ in the space $C^{*}(\bar{\Omega})$ given by the formula

$<f(t), u>=\sum_{x_{i} \epsilon \bar{T}} u\left(x_{i}\right) \omega_{i}(t)+\sum_{x_{j k} \epsilon \bar{T}} u\left(x_{j k}\right) \omega_{j k}(t)$.

Theorem 3. If $t \in \bar{T}, T \in \boldsymbol{T}, \quad$ then the ratios

$$
\begin{gathered}
\|f(t)\|_{C^{*}(\bar{T})} \leq G_{T}, \\
G_{T}=(n+1)\left\{1+\left[\theta_{p q}\left(1-\theta_{p q}\right)\right]^{-1} n / 2\right\},
\end{gathered}
$$

$\operatorname{supp} f(t) \subset \bar{T}, h_{t}(f) \leq \operatorname{diam} T, t \in \bar{T}$

are true.

Proof. Relations (47) follow in an obvious way from (45). Let us turn to the proof of relation (46). Using relations (43) -- (45), we derive

$$
\begin{aligned}
& \quad|<f(t), u>| \leq \max _{x_{s} \in T}\left|u\left(x_{s}\right)\right| \sum_{x_{i}} \in \bar{T}\left|\omega_{i}(t)\right|+ \\
& +\max _{x_{p q} \in \bar{T}}\left|u\left(x_{p q}\right)\right| \sum_{x_{j k} \epsilon \bar{T}}\left|\omega_{j k}(t)\right| \forall t \in T \quad \forall T \epsilon \mathbf{T} .
\end{aligned}
$$

Taking into account (41) -- (42), we find

$$
\begin{aligned}
& \sum_{x_{j k} \in \bar{T}}\left|\omega_{j k}(t)\right|=\sum_{x_{j k} \in \bar{T}} \frac{\lambda_{j}^{T}(t) \lambda_{k}^{T}(t)}{\theta_{j k}\left(1-\theta_{j k}\right)} \leq \\
& \leq \max _{x_{p q} \in \bar{T}}\left[\theta_{p q}\left(1-\theta_{p q}\right)\right]^{-1} \sum_{x_{j k} \in \bar{T}} \lambda_{j}^{T}(t) \lambda_{k}^{T}(t) .
\end{aligned}
$$

By (19) we obtain

$$
\sum_{x_{j k} \in \bar{T}} \lambda_{j}^{T}(t) \lambda_{k}^{T}(t) \leq n(n+1) / 2
$$

so from (49) we find

$\sum_{x_{j k} \epsilon \bar{T}}\left|\omega_{j k}(t)\right| \leq \max _{x_{p q} \in \bar{T}}\left[\theta_{p q}\left(1-\theta_{p q}\right)\right]^{-1} n(n+1) / 2$.

Using (48) -- (50), we have

$$
|<f(t), u>| \leq \max _{t \in \bar{T}}|u(t)|\left\{(n+1)+\max _{x_{p q} \in \bar{T}}\left[\theta_{p q}(1-\right.\right.
$$

$\left.\left.\left.-\theta_{p q}\right)\right]^{-1} n(n+1) / 2\right\} \quad \forall t \in T \quad \forall T \in \mathbf{T}$.

Inequality (46) follows from relation (51). This completes the proof.

Corollary 1. If $\theta_{p q}=1 / 2$ then inequality (46) is simplified:

$$
\|f(t)\|_{C^{*}(\bar{T})} \leq(n+1)(1+2 n) .
$$

Theorem 4. Suppose a function u belongs to the class $C^{3}(\bar{\Omega})$. Let the function $\tilde{u}(t)$ be defined by the formula

$$
\begin{gathered}
\tilde{u}(t)=\sum_{x_{i} \epsilon \bar{T}} u\left(x_{i}\right) \omega_{i}(t)+ \\
+\sum_{x_{j k} \epsilon \bar{T}} u\left(x_{j k}\right) \omega_{j k}(t) \quad \forall t \in T \quad \forall T \in \boldsymbol{T},
\end{gathered}
$$

where $\omega_{i}(t)$ and $\omega_{j k}(t)$ are defined by formulas (39) -(42). Then the inequality

$$
\begin{aligned}
& \tilde{u}(t)-u(t) \mid \leq \\
& \quad \leq G_{T} \sum_{|\alpha|=3}\left\|u^{(\alpha)}\right\|_{C(\bar{T})}(\operatorname{diam} T)^{3} \forall t \in T \quad \forall T \epsilon \boldsymbol{T},
\end{aligned}
$$

is correct.

Formula (54) is obtained in the same way as formula (27) (see Theorem 2).

From (36) -- (42) for any polynomial $P(t)$ of the second degree we have the identity

$$
\begin{gathered}
P(t) \equiv \sum_{x_{i} \epsilon \bar{T}} P\left(x_{i}\right) \omega_{i}(t)+ \\
+\sum_{x_{j k} \epsilon \bar{T}} P\left(x_{j k}\right) \omega_{j k}(t) \quad \forall t \in T \quad \forall T \epsilon \mathbf{T} .
\end{gathered}
$$

Relation (55) is equivalent to the system of identities

$$
\begin{aligned}
t^{\alpha} \equiv \sum_{x_{i} \in \bar{T}} x_{i}^{\alpha} \omega_{i}(t)+ & \\
& +\sum_{x_{j k} \epsilon \bar{T}} x_{j k}^{\alpha} \omega_{j k}(t)
\end{aligned}
$$

$\forall t \in T \quad \forall T \epsilon \mathbf{T} \quad \forall|\alpha| \leq 2$.

By adding information about the support location of the coordinate functions, we obtain:

$\operatorname{supp} \omega_{s}=Z_{s}, \quad \operatorname{supp} \omega_{p q}=Y_{p q}$.

Formulas (56) -- (58) are the approximation relations for Zlamal's approximation.

\section{Conclusion}

In this paper the approach to approximation of the functions defined on the plane region is discussed. The proposed approach allows one to obtain local estimates with the indication of constants. It allows one to take into account changes in the characteristics of the function being approximated (i.e., it allows developing adaptive algorithms). Let us describe some prospects for the development of the proposed approach. Very important objects, often encountered in practice, are functions, the domain of which is a differentiable manifold ( $n$-dimensional sphere, torus, pretzel, projective plane). The problems of mathematical physics about the definition of functions with such domain of definition are encountered in the construction of buildings and structures, as well as in the study of the fundamental properties of matter (using colliders and other devices). The approach proposed in this paper can be applied to rapidly changing functions defined on a manifold. The resulting approximation apparatus is necessary to construct and estimate the rate of convergence 
of methods for solving the above-mentioned problems of mathematical physics. Thus, the development of the proposed approach for rapidly changing functions given on a manifold is a prospect for the development of this approach.

\section{Acknowledgment}

This paper was prepared with the support by a grant from St.Petersburg State University Event 3 (Pure ID 75207094).

\section{References}

[1] J. H. Ahlberg, E. N. Nilson, J. L. Walsh, The Theory of Splines and Their Applications. Mathematics in Science and Engineering: A Series of Monographs and Textbooks, Vol. 38, Academic Press, 1967.

[2] Strang G., Fix G. "Fourier Analysis of the Finite Element Method in Ritz-Galerkin Theory", Stud. Fppl. Math., Vol. 48, N 3, 1969, pp.265-273.

[3] S.G.Michlin, Approximation auf dem Cubischen Gitter. Berlin, 1976.

[4] Philippe Ciarle. The finite element method for elliptic problems, New York, 1978.

[5] S.G.Mikhlin, "Variational-difference approximation", J. Math. Sci., New York 10, 1978, pp.661-787.

[6] S.G.Michlin, Some theorems on the stability of numerical processes, Atti d. Accad. d. Lincei. Classe fis., mat. e nat., 1982, 32, fasc. 2 .

[7] R.S.Varga, Functional Analysis and Approximation Theory in Numerical Analysis, Society for Industrial and Applied Mathematics, 1987.

[8] Yu.K. Dem'yanovich, Local approximations on manifolds and weighted estimates, Journal of Soviet Mathematics, 36 (2), 1987 pp. 261-269.

[9] Yu.K.Dem'yanovich, Local approximation on manifold and minimal splines (monograph), Publishing House of St. Petersburg State University. 1994 (in Russian). ISBN 5-28800481-1.

[10] W. Cai, and J.Wang, “Adaptive Multiresolution Collocation Methods for Initial Boundary Value Problems of Nonlinear PDEs", SIAM J. Num. An., vol. 33, iss. 3, 1996, pp. 937-970. https://www.jstor.org/stable/2158490? seq=1

[11] O.Davydov, G.Nurnberger, “'Interpolation by C1 splines of degree $\mathrm{q}=4$ on triangulations", J. Comput. and Appl. Math., Vol. 126, 2000, pp.159- 183.

[12] Ivo Babuska, Uday Banerjee, John E. Osborn, "Generalized Finite Element Methods: Main Ideas, Results, and Perspective", International Journal of Computational Methods 1 (1), 2004, pp.67-103. DOI:

$10.1142 / \mathrm{S} 0219876204000083$.
[13] J.A. Costa, A.O. Hero, Geodesic entropic graphs for dimension and entropy estimation in manifold, learning, IEEE Trans. Signal Process. 52 (8), 2004, 2210-2221.

[14] Yu.K.Demyanovich, Spline approximations on manifolds, International Journal of Wavelets, Multiresolution and Information Processing, 4 (3), 2006, pp. 383-403.

[15] G.R.Liu, K.Y.Dai, T. T. Nguyen, "A smoothed finite element method for mechanics problems", Comput. Mech. 39, 2007, pp.859 - 877. DOI: 10.1007/s00466-006-0075-4

[16] G.R. Liu, G.R. Zhang, “Edge-based Smoothed Point Interpolation Methods", International Journal of Computational Methods, 5(4), 2008, pp.621-646. DOI: $10.1142 / \mathrm{S} 0219876208001510$

[17] M.-Y. Cheng, H.-t. Wu, Local linear regression on manifolds and its geometric interpretation, J. Amer. Statist. Assoc. 108 (504), 2013, 1421-1434.

[18] Vahid Shobeiri, "'Structural Topology Optimization Based on the Smoothed Finite Element Method", Latin American Journal of Solids and Structures, 13, 2016, pp.378390. DOI: 10.1590/1679-78252243.

[19] Y.K.Dem'yanovich and I. V. Gerasimov, "Local coarsening of simplicial subdivisions", J. Math. Sci., New York 216, No. 2, 2016, 219-236.

[20] W.Zeng, G.R. Liu, "'Smoothed finite element methods (S-FEM): An overview and recent developments", Archives of Computational, Methods in Engineering, 2016. DOI: 10.1007/s11831-016-9202-3.

[21] I.G.Burova,E.F.Muzafarova,I.I.Narbutovskikh, " Local splines of the Second and Third Order, Complex-valued Splines and Image Processing", International Journal of Circuits, Systems and Signal Processing, Vol. 13, 2019, pp.419-429.

[22] B. Sober, D. Levin, Manifold approximation by moving least-squares projection, Constr. Approx., 2019, http://dx.doi.org/10.1007/s00365- 019-09489-8.

[23] H. Saxena, A. Singh, J.N. Rai, “Adaptive spline-based PLL for synchronisation and power quality improvement in distribution system", IET Generation, Transmission and Distribution, 14 (7), 2020, pp. 1311-1319.

[24] R. Amin, S. Nazir, I. Garcia-Magario, “A collocation method for numerical solution of nonlinear delay integrodifferential equations for wireless sensor network and internet of things", Sensors (Switzerland), Vol 20,7, 1962, 2020.

[25] E.H.S. Diop, A.-O. Boudraa, V.B.S Prasath, "Optimal Nonlinear Signal Approximations Based on Piecewise Constant Functions", Circuits, Systems, and Signal Processing, Vol. 39, No 5, 2020, pp. 2673-2694. DOI: 10.1007/s00034-019-01285. 
[26] Z.Yang, H.Liu, T.Bi, Z.Li, Q.Yang, “'An adaptive PMU missing data recovery method", International Journal of Electrical Power and Energy Systems, Vol.116, paper 105577, 2020.

[27] I.G.Burova, Yu.K.Dem'yanovich, T.O.Evdokimova, O.N.Ivancova. Spline-wavelet processing of flows of structured information (monograph), Publishing House of St. Petersburg State University. 2020 (in Russian). ISBN 978-5288-06078-6.
Creative Commons Attribution License 4.0 (Attribution 4.0 International, CC BY 4.0)

This article is published under the terms of the Creative Commons Attribution License 4.0

https://creativecommons.org/licenses/by/4.0/deed.en US 